Article

\title{
Fabrication and Properties of Superhydrophobic Waterborne Polyurethane Composites with Micro-Rough Surface Structure Using Electrostatic Spraying
}

\author{
Fangfang Wang ${ }^{1}$, Lajun Feng ${ }^{1,2, *}$, Guangzhao $\mathrm{Li}^{1}$, Zhe Zhai ${ }^{1}$, Huini Ma ${ }^{1}$, Bo Deng ${ }^{1}$ and \\ Shengchao Zhang ${ }^{3}$ \\ 1 School of Materials Science and Engineering, Xi'an University of Technology, Xi'an 710048, China; \\ wff1170111008@163.com (F.W.); lgz0414@163.com (G.L.); zhaizhe1113@163.com (Z.Z.); \\ 17789276539@163.com (H.M.); dengbo311@163.com (B.D.) \\ 2 Key Laboratory of Corrosion and Protection of Shaanxi Province, Xi'an 710048, China \\ 3 Faculty of Printing, Packing Engineering and Digital Media Technology, Xi'an University of Technology, \\ Xi'an 710048, China; zhangsc1364879367@163.com \\ * Correspondence: fenglajun@xaut.edu.cn; Tel.: +86-029-8231-2733
}

Received: 14 September 2019; Accepted: 21 October 2019; Published: 24 October 2019

\begin{abstract}
Waterborne polyurethane (WPU) coatings hold advantages of good toughness, low cost and environmental protection. However, the low water contact angle (WCA), poor wear and corrosion resistance make them unsuitable for application in the superhydrophobic coatings such as antipollution flashover coatings for transmission lines, self-cleaning coatings for outdoor equipment and waterproof textiles. A series of superhydrophobic WPU composites (SHWPUCs) with micro-rough surface structure was prepared by electrostatic spraying nano-SiO ${ }_{2}$ particles on WPU composites with low surface energy. It showed that as the hydrophobic system content rose the WCAs of the composites first increased and then remained stationary; however, the adhesion and corrosion resistance first increased and then decreased. An appropriate addition of the hydrophobic system content would lead to a dense coating structure, but an excessive addition could increase the interfaces in the coating and then reduce the coating performance. When the mass ratio of the WPU dispersion, polytetrafluoroethylene (PTFE) particles and modified polydimethylsiloxane was 8:0.3:0.4, $10 \mathrm{~g} / \mathrm{m}^{2}$ nano- $\mathrm{SiO}_{2}$ particles were sprayed on the uncured coating surface to construct the SHWPUC with a WCA of $156^{\circ}$. Compared with pure WPU coating, its adhesion and corrosion resistance increased by $12.5 \%$ and one order of magnitude, respectively; its wear rate decreased by $88.8 \%$.
\end{abstract}

Keywords: superhydrophobic WPU composites; electrostatic spraying; nano- $\mathrm{SiO}_{2}$ particles; adhesion; corrosion resistance; wear rate

\section{Introduction}

Protective coatings with superhydrophobicity have a widespread application in the fields of antipollution flashover coatings applied in transmission lines, self-cleaning coatings applied in outdoor equipment and waterproof cloth, etc. [1-3]. However, the most widely used hydrophobic coatings are solvent-borne coatings and their volatile organic solvent (VOC) contents are commonly over $40 \mathrm{wt} \%$. During the processes of production, storage and using, solvent-borne coatings are prone to emit a lot of organic solvents which can not only pollute the environment but also have some potential safety problems [4]. Additionally, these organic solvents are generally flammable and high cost. With the formulation of environmental laws and regulations around the world and the increasing 
environmental protection awareness of human nowadays, it would be inevitable for us to research and apply waterborne coatings holding advantages of low VOC emission, low toxicity and environmental protection. Waterborne polyurethane (WPU) coatings with water as the dispersion medium are basically low cost, solvent-free, non-toxic and non-flammable, in addition, they will not pollute the environment and can avoid safety problems during production and application compared with solvent-based PU coatings [5]. Furthermore, polar groups such as $-\mathrm{COOH}$ and $-\mathrm{OH}$ in the molecular chains of WPU can produce crosslinking polymerization reactions under certain conditions [6-8], thus, it could strengthen the coating structure and enhance the adhesion to the substrates. Due to these excellent properties WPU coatings are extensively applied in many industrial areas such as coatings, adhesives, synthetic leathers, packaging films, membranes, biomaterials and waterproof textiles [9-12].

However, the traditional WPU coatings have some drawbacks such as low water contact angle (WCA), poor wear resistance and corrosion resistance, so that their applications in the superhydrophobic coating are restricted [13]. In order to solve these problems, it is necessary to modify the WPU coating to improve its properties such as hydrophobicity and wear resistance and thus expand its application range [14]. Previous reports have shown that the surface hydrophobicity can be improved by the addition of low surface energy materials on rough surfaces or the construction of rough structures on low surface energy surfaces [15-18]. In recent years, researchers have improved the performances of WPU resins by adding materials containing elements such as fluorine and silicon. Polytetrafluoroethylene (PTFE) material holds excellent properties of high fluoride content, thermochemical stability, high hydrophobicity and low surface friction [19]. Additionally, the WCA of the untreated PTFE coating is up to $120^{\circ}$ and the addition of PTFE materials to the resin matrix can reduce the surface free energy of the coating $[20,21]$. Moreover, the incorporation of nano- $\mathrm{SiO}_{2}$ particles to the coating with low surface energy can not only construct the micro-rough surface structure, increasing the strength of polymer materials, but also improve the wear resistance and corrosion resistance of the coating [22-24]. Shin et al. [25] synthesized a series of waterborne fluorinated acrylate-based PUs for application in antifouling coatings. Krol et al. [26] prepared a kind of hydrophobic WPU coating by the incorporation of fluorine. Serkis et al. [27] prepared a new type of water-based PU/silica nanocomposite by the addition of nano-SiO${ }_{2}$ particles. Zhang et al. [28] used modified nano- $\mathrm{SiO}_{2}$ particles to synthesize UV-curable water-based transparent coating. Generally, the performance of the WPU coating has been improved to some extent by modification, but the process is complex and time-consuming, moreover, the dispersion of nano-fillers has not been well dissolved. Therefore, the method of electrostatic spraying nano- $\mathrm{SiO}_{2}$ particles was used in this work to construct micro-rough surface structure on the coating with low surface energy and to improve the properties such as hydrophobicity and wear resistance. Electrostatic spraying is pollution-free and has excellent atomization capability and operability. In addition, nano- $\mathrm{SiO}_{2}$ particles can be well dispersed under the action of a high-voltage electrostatic field and most importantly, the driving force arising from the compressed air may make the nanoparticles greatly adhere to the coating surface [29].

The modified PTFE particles with different contents were added to the WPU dispersions to prepare WPU composites with low surface energy (LSWPUCs). Then, nano-SiO ${ }_{2}$ particles were sprayed using an electrostatic spraying device on the surfaces of the uncured LSWPUCs to form a micro-rough surface in this work. Thus, the superhydrophobic WPU composites (SHWPUCs) were formed owing to the adhesion of nano- $\mathrm{SiO}_{2}$ particles in the coating during curing. The adhesion, corrosion resistance and wear resistance of the obtained SHWPUCs were measured to evaluate their durability. Additionally, the effects of the micro-rough surface structure and the hydrophobic system content on the properties of WPU composites were studied. This may provide a useful reference for the design, preparation and application of superhydrophobic waterborne coatings. 


\section{Materials and Methods}

\subsection{Experimental Materials}

WPU dispersion was supplied by Jining Huakai Resin Co. Ltd. (Jining, China). Its solid content, viscosity and VOC concentration were $35 \%, 75 \mathrm{cps}$ and $253 \mathrm{~g} / \mathrm{L}$, respectively. Polydimethylsiloxane (PDMS) emulsion (average $M_{\mathrm{w}}=115,000 \mathrm{~g} \mathrm{~mol}^{-1}$ ) was supplied by Shanghai Yuanye Biotechnology Co. Ltd. (Shanghai, China). Ethyl silicate (TEOS, analytically pure) was supplied by Tianjin Kermio Chemical Reagent Co. Ltd. (Tianjin, China). Dioctyldilauryltin (DOTDL, $M_{\mathrm{w}}=743.7 \mathrm{~g} \mathrm{~mol}^{-1}$ ), 98\%, was supplied by Shanghai Yuanye Biotechnology Co. Ltd. (Shanghai, China). Sodium chloride ( $\mathrm{NaCl}$, analytically pure) was supplied by Tianjin Kemio Chemical Reagent $\mathrm{Co}$. Ltd. (Tianjin, China). Anhydrous ethanol (analytically pure) was supplied by Tian in Fuyu Fine Chemical Co. Ltd. (Tianjin, China). PTFE particles (MP1200) particles with average particle size $\leq 3 \mu \mathrm{m}$ were purchased from Dupont (Wilmington, DE, US). Nano-SiO 2 (N-100) particles were supplied by Jining Huakai Resin Co. Ltd. (Jining, China). The average diameter, specific surface area, and silica content of nano- $\mathrm{SiO}_{2}$ were respectively $40-60 \mathrm{~nm}, 300 \pm 25 \mathrm{~m}^{2} / \mathrm{g}$ and $99.8 \%$, respectively.

Q235 steel $(50 \mathrm{~mm} \times 20 \mathrm{~mm} \times 3 \mathrm{~mm})$ was used as the metal substrate and was roughened by a YX-6050A sand blasting device (Anbangruiyuxin Machine Technology Development Co. Ltd., Wuhan, China). The air pressure was 0.6-0.8 MPa. The distance between the Q235 steel substrate and the spray gun was $110-150 \mathrm{~mm}$. The spray time was $30-40 \mathrm{~s}$.

\subsection{Preparation of the LSWPUCS}

PTFE particles should be modified to improve the dispersibility before adding. At room temperature, PDMS, TEOS (5 wt \% vs. PDMS) and DOTDL (1.5 wt \% vs. PDMS) were mixed by an 85-2 magnetic stirring device (Hangzhou Instrument Motor Co., Ltd., Hangzhou, China) at a speed of 100-150 r/min for $30 \mathrm{~min}$. Then, the mixture was treated by a KQ-50B ultrasonic dispersion device (Kunshan Ultrasonic Instrument Co., Ltd., Kunshan, China) for $10 \mathrm{~min}$ to prepare the modified PDMS (M-PDMS) emulsion (shown in Figure 1A). Subsequently, the M-PDMS emulsion and PTFE particles were added to WPU by magnetic stirring at a speed of $250-300 \mathrm{r} / \mathrm{min}$ for $40 \mathrm{~min}$. The obtained M-PDMS/PTFE/WPU dispersions (shown in Table 1) were first placed at room temperature for 20-30 min and then coated on the Q235 steel substrates. The thickness of the coatings $(110-120 \mathrm{~mm})$ was controlled by weighting during coating. After coating, the samples were cured first at room temperature for 1 day and then at $150{ }^{\circ} \mathrm{C}$ for $1 \mathrm{~h}$ in an oven (Zhejiang Yuyao Yuandong CNC Instrument Factory, Yuyao, China). Thus, a layer of the LSWPUC was prepared on the Q235 steel substrate. The samples were named as \#1 LSWPUC, \#2 LSWPUC, \#3 LSWPUC and \#4 LSWPUC. In addition, the pure WPU coating (named as \#0) was also cured in the same way described previously for reference.

Table 1. System of the modified polydimethylsiloxane (M-PDMS)/polytetrafluoroethylene (PTFE)/waterborne polyurethane (WPU) dispersions.

\begin{tabular}{ccccc}
\hline Samples & \#1 & \#2 & \#3 & \#4 \\
\hline WPU (g) & 6 & 8 & 8 & 6 \\
PTFE (g) & 0.1 & 0.2 & 0.3 & 0.3 \\
M-PDMS (g) & 0.2 & 0.2 & 0.4 & 0.6 \\
\hline
\end{tabular}

\subsection{Preparation of the SHWPUCS}

The M-PDMS/PTFE/WPU dispersions were prepared and then coated on the Q235 steel substrates in the same way described previously. After coating, the samples were placed at room temperature for 2-3 h. In this case, the obtained coatings were under semi-dry and nonflowing conditions and their surfaces were slightly tacky. In order to enhance the dispersion and adhesion of nano-SiO particles, a NEW KCI-CU801 electrostatic spraying device (Shenzhen Honghaida Instrument Co., 
Ltd., Shenzhen, China) was used to spray $\mathrm{SiO}_{2}$ nanoparticles on the uncured coating surfaces to construct the micro-rough surface structure. The sprayed content of nano- $\mathrm{SiO}_{2}$ particles was $10 \mathrm{~g} / \mathrm{m}^{2}$. The voltage of electrostatic spraying was $50-60 \mathrm{kV}$. The pressure of the compressed air was $0.6-0.7 \mathrm{MPa}$. The spraying time was 20-30 s. The distance between the spray gun and the sample was $110-150 \mathrm{~mm}$. After spraying, the samples were cured first at room temperature for 1 day and then at $150{ }^{\circ} \mathrm{C}$ for $1 \mathrm{~h}$ in an oven. Thus, a layer of the SHWPUC was completely prepared on the Q235 steel substrate. The schematic illustration of the LSWPUC is shown in Figure 1. The obtained samples were named as \#1 SHWPUC, \#2 SHWPUC, \#3 SHWPUC and \#4 SHWPUC.

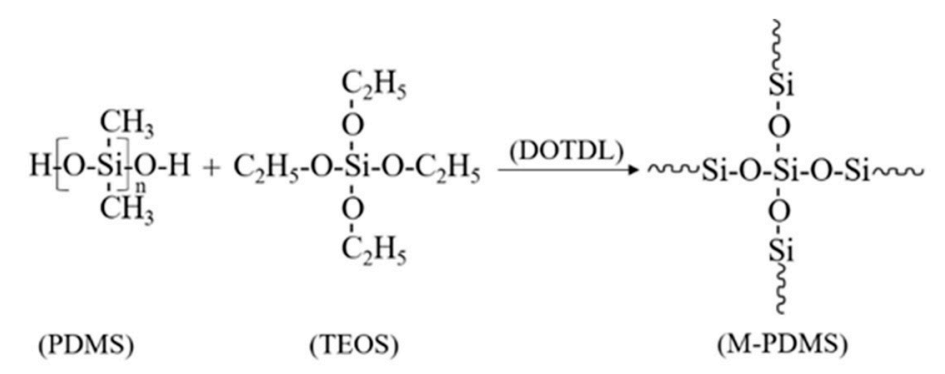

(B)
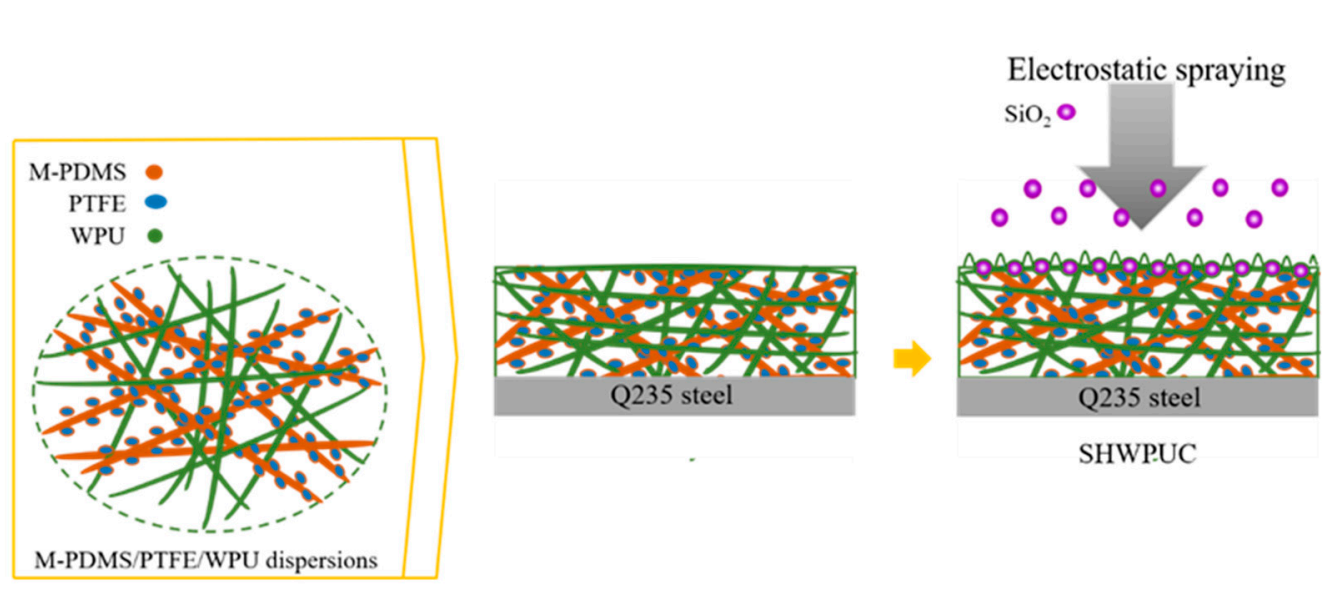

Figure 1. (A) modification of the PDMS emulsion and (B) Schematic illustration of the superhydrophobic WPU composite (SHWPUC).

\subsection{Measurements}

\subsubsection{WCA Test}

The WCAs of the coatings were measured by an SDC-200 contact angle testing machine (Dongguan Shengding Precision Instrument Co., Ltd., Guangzhou, China) to characterize the hydrophobicity. A $20 \mu \mathrm{L}$ pure water droplet was injected with a micro syringe for each test. Each of the two samples for each coating was measured six times and the measurements were averaged to determine the WCA.

\subsubsection{Adhesion Test}

The adhesion test was performed according to ISO 4624:1978 at room temperature by a D2-5DL universal mechanical testing machine (Changchun Mechanical Institute, Changchun, China). The coating was totally peeled off from the steel substrate during test. Each coating was measured ten times and the measurements were averaged to calculate the adhesion by Equation (1):

$$
\sigma=P / A,
$$

where $\sigma$ is the adhesion (MP), $P$ is the maximum load $(\mathrm{N})$ and $A$ is the coating area $\left(\mathrm{mm}^{2}\right)$. 


\subsubsection{Corrosion Resistance Test}

A $3.5 \mathrm{wt} \% \mathrm{NaCl}$ solution was used as the corrosion medium to test the corrosion resistance of the obtained coatings under harsh conditions. The polarization curve and electrochemical impedance spectroscopy of the coating were measured at room temperature by a Ver4.2corr Test System (Wuhan Corr Test Co., Ltd., Wuhan, China) with a three-electrode cell. The sample was used as the working electrode, the Pt electrode was used as the auxiliary electrode, and the saturated calomel electrode was used as the reference electrode. The test area was $0.785 \mathrm{~cm}^{2}$. The samples were immersed in $3.5 \mathrm{wt} \% \mathrm{NaCl}$ solution at $40{ }^{\circ} \mathrm{C}$ for 30 days before testing. The testing was started when the open circuit potential of the system was stable.

\subsubsection{Wear Resistance Test}

The wear resistance test of the coating was carried out at room temperature in accordance with ASTM G99-05 by an HT-1000 high temperature scratch testing machine (Lanzhou Zhongke Kaihua Development Co., Ltd., Lanzhou, China), with the coating against a steel bearing ball (Ø2.5 mm) with a hardness level of HRC62. The applied load was $5 \mathrm{~N}$, the rotation speed of the steel ball was $400 \mathrm{r} / \mathrm{min}$, the sliding radius was $5 \mathrm{~mm}$, and the wear time was $10 \mathrm{~min}$. The wear rate and friction coefficient were used to evaluate the wear resistance of the coating. The wear rate was calculated by Equation (2).

$$
I=\Delta m / 2 \pi r n t F \varrho,
$$

where $I$ is the specific wear rate $\left(\mathrm{cm}^{3} / \mathrm{mm} \mathrm{N}\right), \Delta m$ is the loss weight $(\mathrm{g}), r$ is the sliding radius $(\mathrm{mm}), n$ is the rotation speed of the steel ball $(\mathrm{r} / \mathrm{min}), t$ is the wear time $(\mathrm{min}), F$ is the applied load $(\mathrm{N})$ and $\varrho$ is the density of the WPU coating $\left(\mathrm{g} / \mathrm{cm}^{3}\right)$.

\subsubsection{Morphology Analysis}

The 3D micromorphologies of the coating surfaces were observed by an LEXT OLS4000 laser confocal scanning microscope (OLYMPUS, Tokyo, Japan) to characterize the effect of the micro-rough surface structure on the hydrophobicity of the coatings. The magnification was 108-17,280x; the field of view was $16 \mu \mathrm{m}$ to $2.56 \mathrm{~mm}$.

After the wear test, the surface morphologies of the wear tracks were observed by a VEGA3 XMU Scanning Electron Microscope (SEM) (TESCAN, Brno, Czech).

\section{Results and Discussion}

\subsection{Hydrophobicity of WPU Composites}

Figure 2 shows the WCAs of different WPU composites. The WCAs of \#0 (pure WPU coating), \#1 LSWPUC, \#2 LSWPUC, \#3 LSWPUC and \#4 LSWPUC were $83^{\circ}, 107^{\circ}, 110^{\circ}, 112^{\circ}$ and $113^{\circ}$, respectively, stating that the low surface energy coating was successfully prepared by the incorporation of the hydrophobic system of M-PDMS/PTFE. When the M-PDMS/PTFE system content was below 8.05 wt \% (\#3 M-PDMS/PTFE system vs. M-PDMS/PTFE/WPU system), the WCAs of the LSWPUCs first increased and then remained stationary as the M-PDMS/PTFE system content rose. The reason may be as follows. PTFE as a kind of symmetrical and non-polar polymer has high fluoride content and low surface energy. PDMS holds low surface energy and the M-PDMS will generate a crosslinked network structure after modification. Then, the M-PDMS as the modifier in this work would adsorb on the surfaces of PTFE particles. Under the action of magnetic stirring, the M-PDMS/PTFE system could be evenly dispersed in the WPU dispersion, leading to the reduction of the surface energy of the WPU composites. When the M-PDMS/PTFE system content reached $8.05 \mathrm{wt} \%$, the hydrophobic groups of the coating surface were under the saturated condition. As the M-PDMS/PTFE system content continued to increase, the change in the WCA of the LSWPUC remained stable. 
It can be seen from Figure 2 that the WCAs of \#1 SHWPUC, \#2 SHWPUC, \#3 SHWPUC and \#4 SHWPUC were $154^{\circ}, 155^{\circ}, 156^{\circ}$ and $155^{\circ}$, respectively, displaying their superhydrophobic behaviors. This indicated that electrostatic spraying nano- $\mathrm{SiO}_{2}$ particles on the coating surface with low surface energy could construct the micro-rough surface structure and then improve the hydrophobicity of the coating. This may be explained by the following. The WPU composites were all in the uncured state when nanoparticles were sprayed. Under the action of high-voltage electrostatic field, nano-SiO particles would first enter the locations of the coatings where there were some defects or less nano- $\mathrm{SiO}_{2}$ particles and thus result in an even distribution. In addition, nano- $\mathrm{SiO}_{2}$ particles can be rushed into the uncured composites due to the high air pressure of electrostatic spraying. Subsequently, a layer of uniform WPU composites could be coated on the surfaces of nano- $\mathrm{SiO}_{2}$ particles [23]. Therefore, the micro-rough surface structure was formed on the surfaces of the WPU composites, as shown in Figure 1.

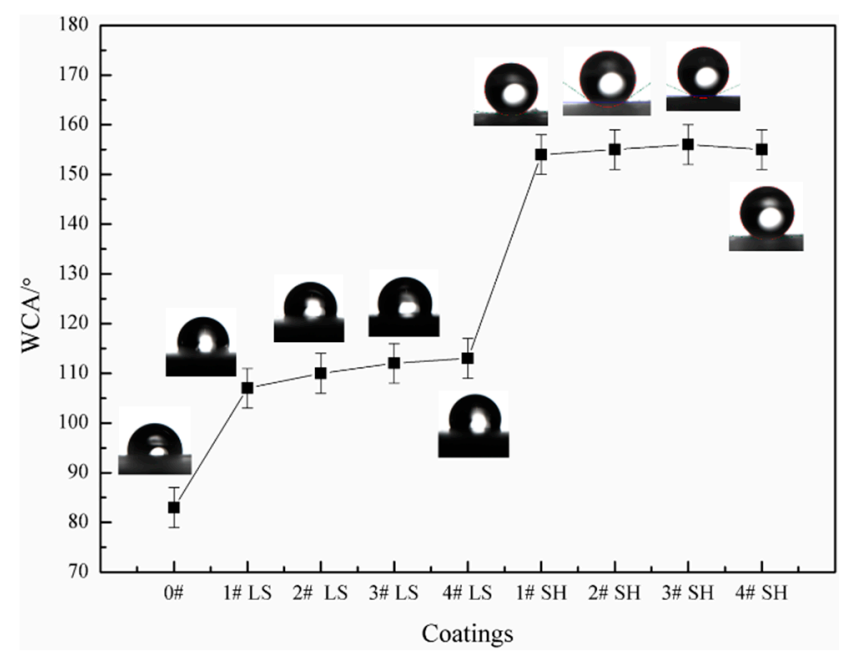

Figure 2. Water contact angles (WCAs) of the WPU composites with different M-PDMS/PTFE system contents.

Figure 3 shows the 3D micromorphologies of different coating surfaces as observed by laser confocal scanning microscope. The surface micromorphology of \#0 (Figure 3A) was as smooth as that of \#3 LSWPUC (Figure 3B), indicating that the incorporation of M-PDMS/PTFE system would not greatly affect the surface micromorphologies of the prepared WPU composites. The micro-rough surface structure on the surface of \#3 SHWPUC (Figure 3C) was similar to that of \#4 SHWPUC (Figure 3D). However, due to the facts that a low content of the M-PDMS/PTFE system was added to \#3 SHWPUC and that the number of nano- $\mathrm{SiO}_{2}$ particles embedded in its surface was relatively uniformly compact, the resulting micro-rough structure of \#3 SHWPUC was a little better than that of \#4 SHWPUC. This result was consistent with that of the WCA tests. Moreover, it demonstrated that the hydrophobicity of the coating may be closely related to the micro-rough surface structure and the superhydrophobic coating can be prepared by electrostatic spraying [30]. 

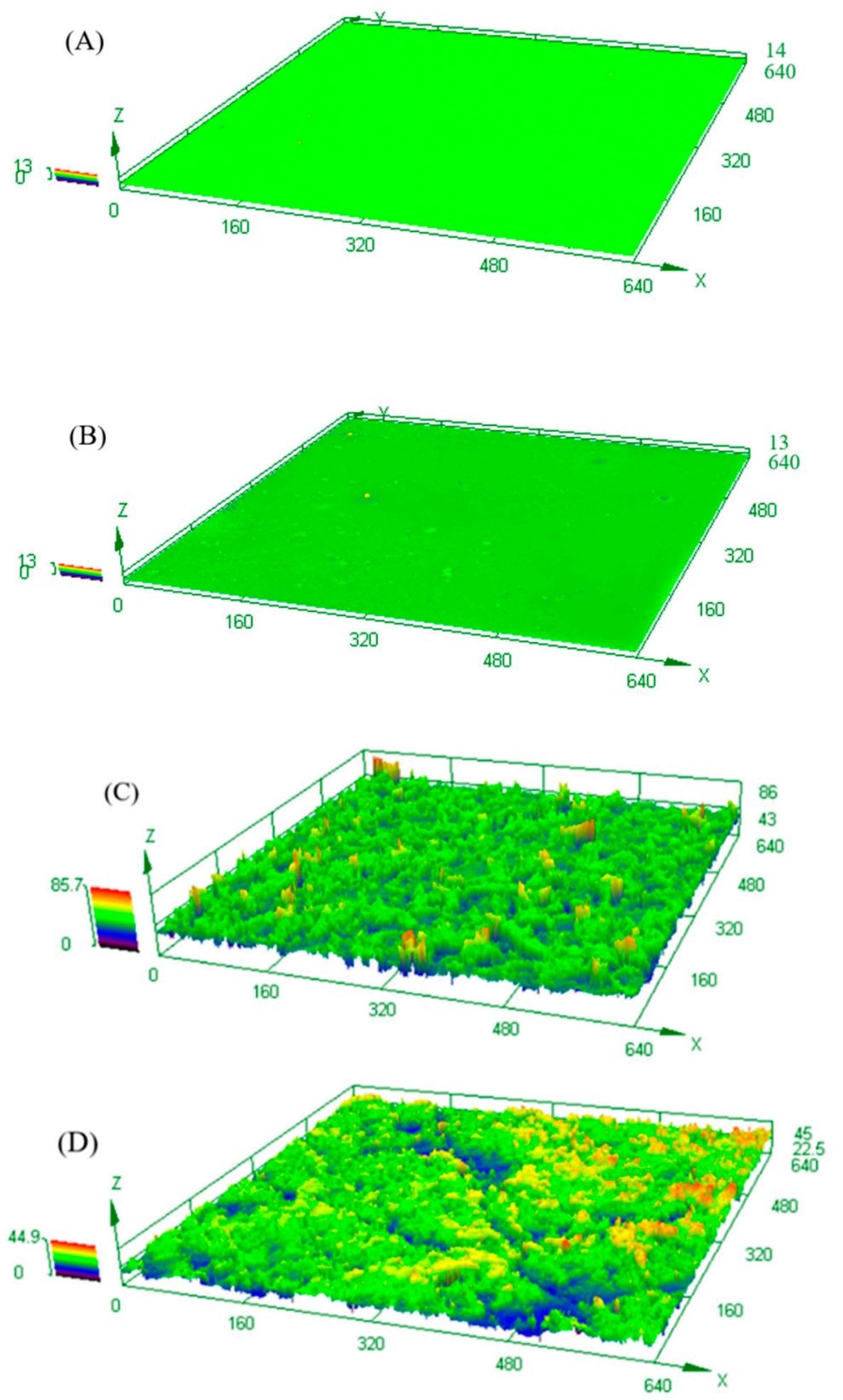

Figure 3. Micromorphologies of different WPU composites of (A) \#0; (B)\#3 LSWPUC; (C)\#3 SHWPUC; and (D) \#4 SHWPUC, $(\mu \mathrm{m})$.

According to the Wenzel equation, $\cos \theta_{\omega}=r \cos \theta_{0}$, where $\theta_{\omega}$ is the apparent contact angle, $\theta_{0}$ is the eigen contact angle and $r$ is the micro-roughness. When $\theta_{0}$ of the coating was over $90^{\circ}$, its hydrophobicity increased as the surface micro-roughness rose. It can be seen that the LSWPUCs prepared by the addition of the M-PDMS/PTFE system to WPU were hydrophobic; their WCAs were over $90^{\circ}$. In addition, spraying nano- $\mathrm{SiO}_{2}$ particles on the coating surfaces increased the micro-roughness and obtained the SHWPUCs whose WCAs were greater than $150^{\circ}$.

In the case of Cassie-Baxter state, the rough surface is regarded as a compound interface composed of air and solid. Water droplets can stand at the top of the rough structure of the superhydrophobic surface which has very low adhesion to water droplets. According to Cassie-Baxter equation, $\cos \theta_{c}=f\left(1+\cos \theta_{0}\right)-1$, where $\theta_{c}$ is the apparent contact angle, $\theta_{0}$ is the eigen contact angle, and $f$ is the solid-liquid contact area percent. $\theta_{c}$ can increase with the decrease of $f$. As for \#3 SHWPUC, its $\theta_{0}$ and $\theta_{c}$ were $112^{\circ}$ and $156^{\circ}$, respectively, and it was calculated that $f=0.13$. That is, the solid-gas 
contact area percent reached $87 \%$, indicating that a large amount of gas was between the micro-rough structures of the coating surface and thus the coating was superhydrophobic.

\subsection{Adhesion of the SHWPUCS}

The adhesion of the coating to the steel substrate is a main performance evaluation. Figure 4 shows the adhesion of different SHWPUCs to the steel substrates. With an increase in the M-PDMS/PTFE system content, the adhesion of the SHWPUC to the Q235 steel substrate first increased and then declined. The adhesions of \#0, \#1 SHWPUC, \#2 SHWPUC, \#3 SHWPUC and \#4 SHWPUC were 3.26, $3.34,3.42,3.67$ and $3.11 \mathrm{MPa}$, respectively. The adhesion of \#3 SHWPUC was the best among all the obtained coatings, which increased by $12.58 \%$ compared with that of \#0. The reason may be that the addition of a small number of PTFE particles to the WPU resins could fill the pores arising from the water evaporation during curing of WPU and then the contact areas between the WPU resin and the metal substrate could increase. Thus, the adhesion of the coating would be strengthened. Additionally, the pores of \#3 SHWPUC were basically filled when the M-PDMS/PTFE system content reached the critical value of $8.05 \mathrm{wt} \%$, so that the coating structure became dense and the adhesion to the steel substrate was enhanced. As the M-PDMS/PTFE system content went on to rise, the excessive PTFE particles would occupy too much contact area between the WPU resins and the metal substrate, leading to the reduction of the adhesion of \#4 SHWPUC.

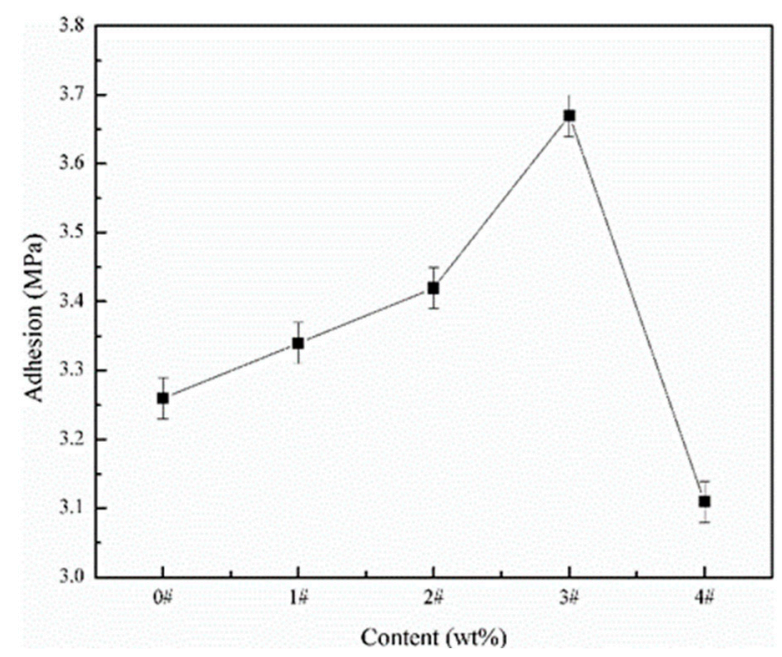

Figure 4. Adhesion of different SHWPUCs to the steel substrates.

\subsection{Corrosion Resistance of the SHWPUCs}

Figure 5A shows polarization curves of the SHWPUCs with different M-PDMS/PTFE system contents after an immersion in $3.5 \mathrm{wt} \% \mathrm{NaCl}$ solution at $40{ }^{\circ} \mathrm{C}$ for 30 days. Figure $5 \mathrm{~B}$ shows corrosion rates of different SHWPUCs which were calculated by computer software. The corrosion rates of \#0, \#1 SHWPUC, \#3 SHWPUC and \#4 SHWPUC were $2.21 \times 10^{-2}, 1.11 \times 10^{-2}, 0.29 \times 10^{-2}$ and $2.06 \times 10^{-2} \mathrm{~mm} / \mathrm{a}$, respectively. As the M-PDMS/PTFE system content increased the corrosion resistance of the SHWPUC first increased and then declined. The facts may be as follows. When the M-PDMS/PTFE system content was low, PTFE particles could fill the pores generated by the water evaporation of the WPU coating during curing. Then, the coating structure would become relatively compact to prevent the diffusion of the corrosive medium. Thus, the corrosion resistance of the coating would be improved. However, with M-PDMS/PTFE system content over $8.05 \mathrm{wt} \%$, the increased interfaces between PTFE particles and WPU resins could increase the diffusion ability of the corrosive medium and thus reduce the corrosion resistance of the coating. The corrosion rate of \#3 SHWPUC was about one order of magnitude lower than that of \#0. The coating structure and corrosion resistance of \#3 SHWPUC were the best among all the obtained coatings. 

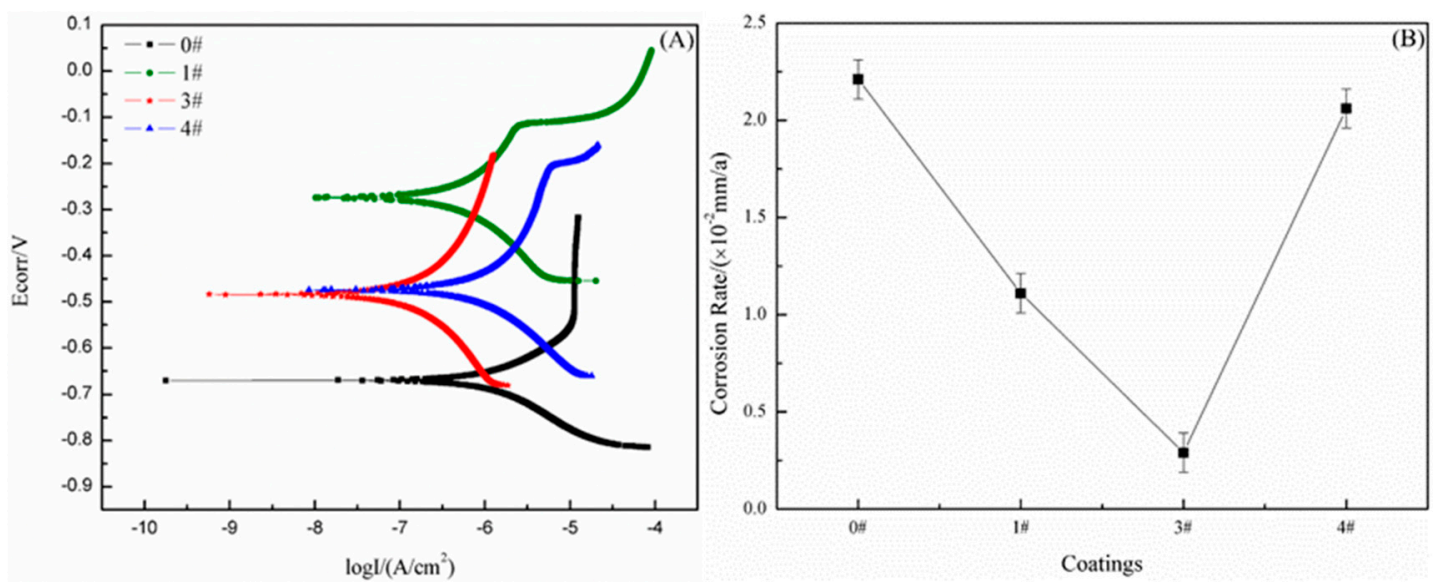

Figure 5. (A) Polarization curves and (B) corrosion rates of different SHWPUCs immersed in 3.5 wt \% $\mathrm{NaCl}$ solution at $40^{\circ} \mathrm{C}$ for 30 days.

Figure 6A shows Nyquist plots of the SHWPUCs with different M-PDMS/PTFE system contents immersed in $3.5 \mathrm{wt} \% \mathrm{NaCl}$ solution at $40{ }^{\circ} \mathrm{C}$ for 30 days. A nearly complete semicircle was observed in the high frequency range on the Nyquist plots and the semicircle diameter showed the insulation and shielding properties of the coating [29]. As the M-PDMS/PTFE system content increased, the semicircle diameter in the high-frequency range of the SHWPUC first increased and then decreased. The semicircle diameters in the high frequency range of \#1 SHWPUC, \#3 SHWPUC and \#4 SHWPUC were all larger than that of \#0. Furthermore, the largest semicircle diameter in the high frequency region of \#3 SHWPUC would hold the best shielding performance against the corrosive medium.
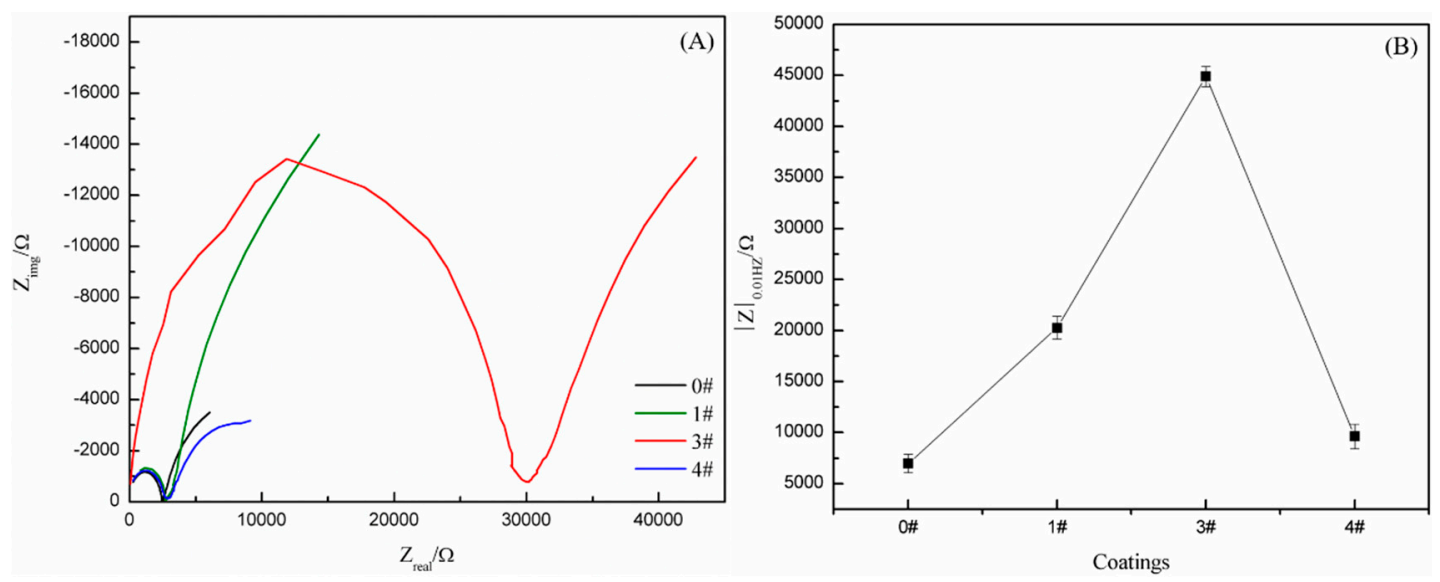

Figure 6. (A) Nyquist plots and (B) $|\mathrm{Z}|_{0.01 \mathrm{~Hz}}$ values of different SHWPUCs after an immersion in $3.5 \mathrm{wt}$ $\% \mathrm{NaCl}$ solution at $40^{\circ} \mathrm{C}$ for 30 days.

A quarter semicircle in the low frequency range was related to the corrosion reaction between the electrolyte and the metal substrate and clearly stated that the $\mathrm{NaCl}$ solution had penetrated to the Q235 steel substrate after an immersion for 30 days. Figure $6 \mathrm{~B}$ shows the $|\mathrm{Z}|_{0.01 \mathrm{~Hz}}$ values of the SHWPUCs with different M-PDMS/PTFE system contents. The $|Z|_{0.01 \mathrm{~Hz}}$ value of the SHWPUC first increased and then decreased with the increase of the M-PDMS/PTFE system content. The $|\mathrm{Z}|_{0.01 \mathrm{~Hz}}$ values of \#0, \#1 SHWPUC, \#3 SHWPUC and \#4 SHWPUC were 6,988.47, 20,267.16, 44,888.46 and 9,618.51, respectively. A higher $|\mathrm{Z}|_{0.01 \mathrm{~Hz}}$ value of the coating in the low frequency range indicated that the coating may have a stronger shielding effect on the external corrosive medium. The $|Z|_{0.01 \mathrm{~Hz}}$ value of \#3 SHWPUC was the highest among all the obtained coatings, which was $75.24 \%$ higher than that of \#0. The result further indicated that the coating structure of \#3 SHWPUC was the densest among all coatings. 


\subsection{Wear Resistance of the SHWPUCS}

It is evident that the hydrophobicity and corrosion resistance of the obtained \#3 SHWPUC were the best among all WPU composites. Therefore, the wear resistance of \#3 SHWPUC was further investigated and it was compared with that of the pure WPU coating under same conditions. During the wear test, the applied load was $5 \mathrm{~N}$, the rotation speed of the steel ball was $400 \mathrm{r} / \mathrm{min}$, the sliding radius was $5 \mathrm{~mm}$ and the wear time was $10 \mathrm{~min}$. Figure $7 \mathrm{~A}$ shows the friction coefficient-time curves of $\# 0$ and \#3 SHWPUC, and Figure 7B shows their wear rates. The friction coefficient-time curve of \#0 was uniformly wavy, however, the friction coefficient-time curve of \#3 SHWPUC first greatly fluctuated and then became smooth after $6 \mathrm{~min}$. This may be ascribed to the low surface friction of the fluorine element [24]. The wear rate $\left(1.33 \times 10^{-10} \mathrm{~cm}^{3} / \mathrm{mm} \mathrm{N}\right)$ and friction coefficient $(0.08)$ of \#3 SHWPUC declined by $88.82 \%$ and $78.38 \%$, respectively, compared with those of $\# 0,1.19 \times 10^{-9} \mathrm{~cm}^{3} / \mathrm{mm} \mathrm{N}$ and 0.37. The reason for this would be that under the action of a high-voltage electrostatic field the surface activity of nano- $\mathrm{SiO}_{2}$ particles was probably enhanced and then polar groups such as $-\mathrm{OH}$ groups were absorbed on the surfaces of nano- $\mathrm{SiO}_{2}$ particles. Thus, the polymerization reaction that occurred between these polar groups and some polar groups in the molecular structure of WPU during curing made nano- $\mathrm{SiO}_{2}$ firmly embed to the composite coating, so that it was difficult for nano- $\mathrm{SiO}_{2}$ particles to wear off. In addition, the high hardness and strength of nano- $\mathrm{SiO}_{2}$ particles could improve the strength of \#3 SHWPUC, whose wear resistance was better than that of \#0.
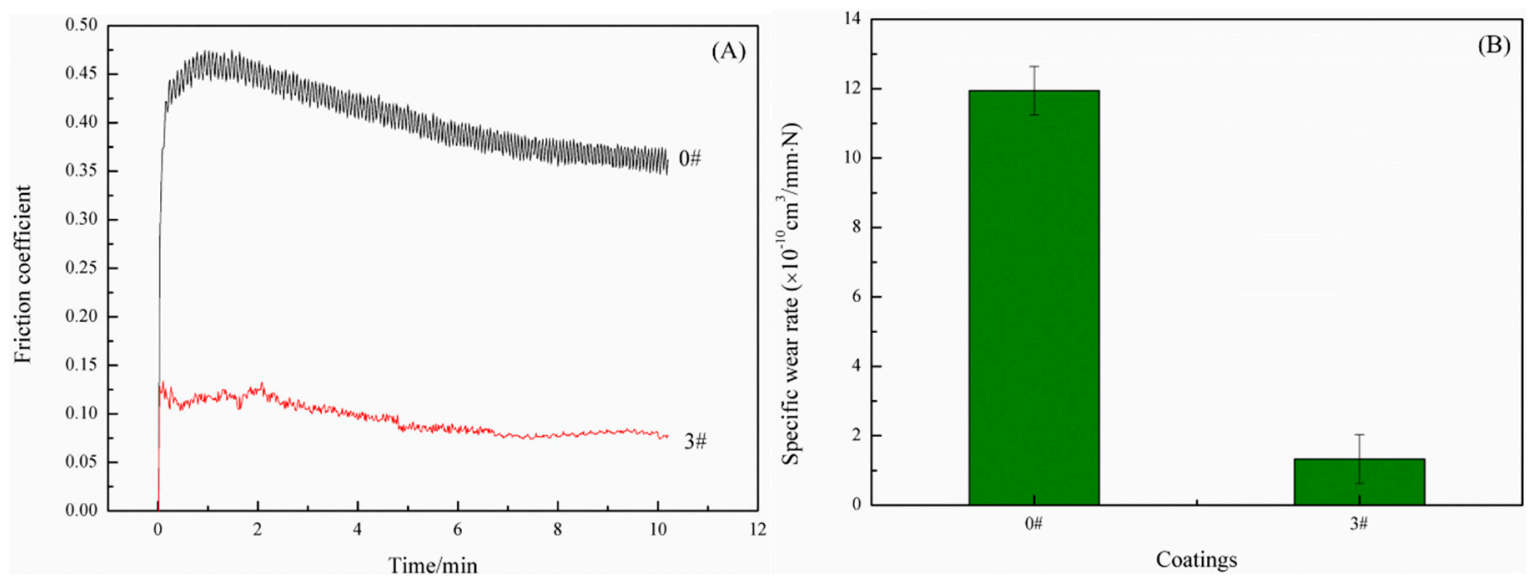

Figure 7. (A) Friction coefficient-time curves and (B) wear rates of \#0 and \#3 SHWPUC.

Figure 8 shows the wear track morphologies of $\# 0$ and \#3 SHWPUC after the wear test. There were a few worn pieces on the wear track of \#0 (Figure 8A), which would become abrasive materials to exacerbate the wear of the coating. The wear rate and friction coefficient of \#0 were aggravated and a wavy friction coefficient-time curve was generated. It is obvious that there were almost no block pieces on the wear track of \#3 SHWPUC and its wear track seemed to be shallow (Figure 8B). EDS shows that there were a large number of Si and F elements in the wear track of \#3 SHWPUC (Figure 8C), which may have reduced its friction coefficient and wear rate and thus improved its wear resistance. This further indicated that the superhydrophobic surface of the coating with excellent wear resistance can be constructed by electrostatic spraying. 

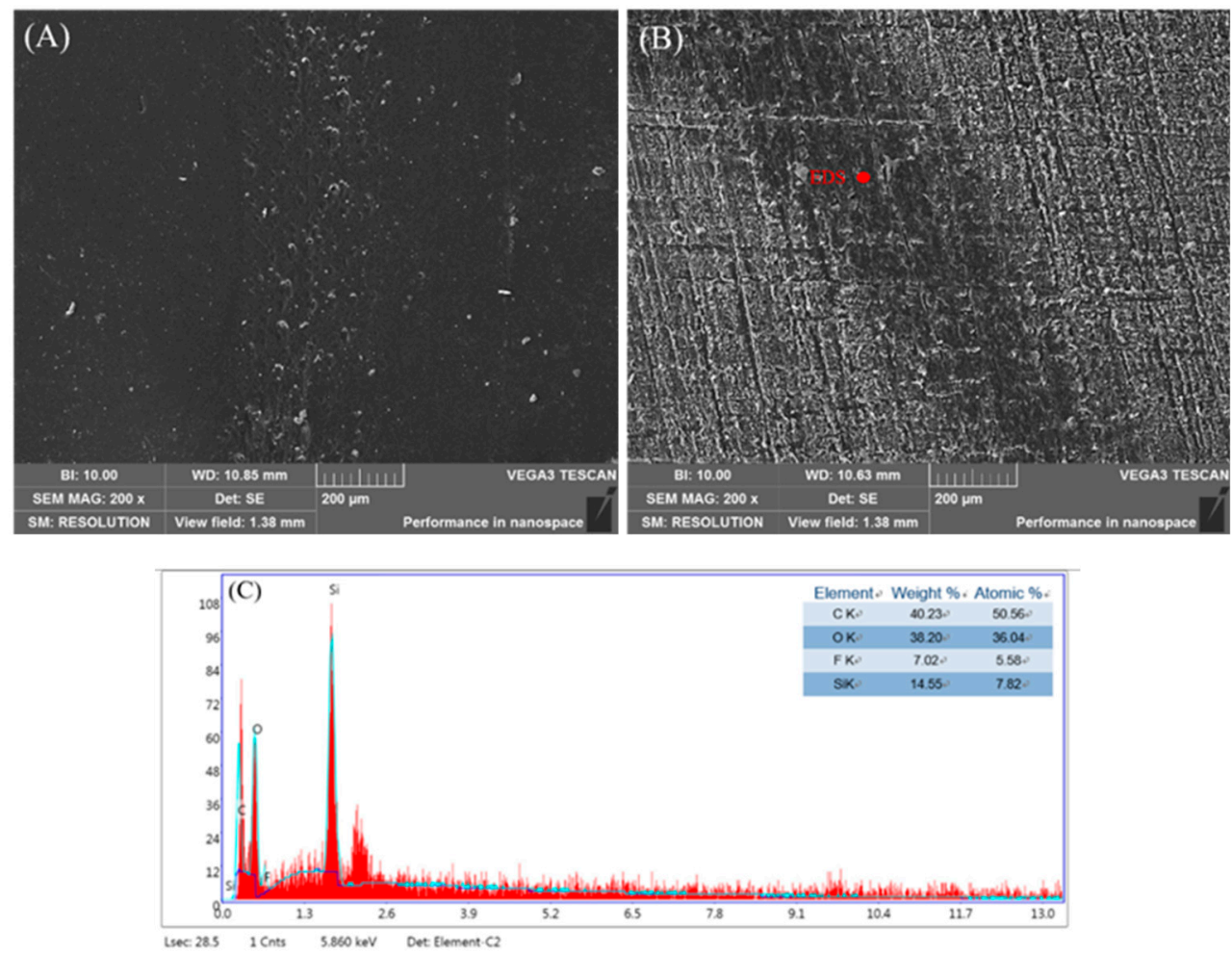

Figure 8. Wear track morphologies of (A) \#0; and (B) \#3 SHWPUC. (C) EDS of \#3 SHWPUC.

\section{Conclusions}

A series of superhydrophobic WPU composites with micro-rough surface structure were prepared by electrostatic spraying nano-SiO $\mathrm{S}_{2}$ particles on the obtained WPU composites with low surface energy. It was concluded that the hydrophobic WPU composite was prepared by adding the M-PDMS/PTFE system to the WPU dispersion. As the M-PDMS/PTFE system content rose, the WCAs of the composites first increased and then remained stationary; however, the adhesion and corrosion resistance first increased and then decreased. An appropriate addition of the hydrophobic system content would lead to a dense coating structure, but an excessive addition could increase the interfaces in the coating and then reduce the coating performance. When the mass ratio of the WPU dispersion, PTFE particles and M-PDMS was 8:0.3:0.4, $10 \mathrm{~g} / \mathrm{m}^{2}$ nano-SiO 2 particles were sprayed on the uncured coating surface to construct the \#3 SHWPUC, whose WCA was $156^{\circ}$. Compared with the pure WPU coating, its adhesion $(3.67 \mathrm{MPa})$ increased by $12.5 \%$, its corrosion rate $\left(0.29 \times 10^{-2} \mathrm{~mm} / \mathrm{a}\right)$ was reduced by almost one order of magnitude, and moreover, its wear rate $\left(1.33 \times 10^{-10} \mathrm{~cm}^{3} / \mathrm{mm} \mathrm{N}\right)$ and friction coefficient $(0.08)$ decreased by $88.8 \%$ and $78.3 \%$, respectively.

Author Contributions: Conceptualization, L.F. and F.W.; methodology, Z.Z., B.D., H.M. and S.Z.; writing一original draft preparation, F.W.; writing—review and editing, L.F. and F.W.; supervision, G.L.

Funding: This work was supported by the Natural Science Foundation of Shaanxi Province, China, grant number 2018JM5053.

Conflicts of Interest: The authors declare no conflict of interest.

\section{References}

1. Shang, Y.; Si, Y.; Raza, A.; Yang, L.; Mao, X.; Ding, B.; Yu, J. An in situ polymerization approach for the synthesis of superhydrophobic and superoleophilic nanofibrous membranes for oil-water separation. Nanoscale 2012, 4, 7847-7854. [CrossRef] [PubMed] 
2. Yokoi, N.; Manabe, K.; Tenjimbayashi, M.; Shiratori, S. Optically transparent superhydrophobic surfaces with enhanced mechanical abrasion resistance enabled by mesh structure. ACS Appl. Mater. Inter. 2015, 7, 4809-4816. [CrossRef] [PubMed]

3. Mukhopadhyay, A.; Midha, V.K. A review on designing the waterproof-breathable fabrics Part I: Fundamental principles and designing aspects of breathable fabrics. J. Ind. Text. 2008, 37, 225-262. [CrossRef]

4. Solouki Bonab, V.; Manas-Zloczower, I. Chemorheology of thermoplastic polyurethane and thermoplastic polyurethane/carbon nanotube composite systems. Polymers 2016, 99, 513-520. [CrossRef]

5. Yang, P.; Wang, Y.; Lu, L.; Yu, X.; Liu, L. Surface hydrophobic modification of polyurethanes by diaryl carbene chemistry: Synthesis and characterization. Appl. Surf. Sci. 2018, 435, 346-351. [CrossRef]

6. Arukula, R.; Narayan, R.; Sreedhar, B.; Rao, C.R.K. High corrosion resistant-redox active-moisture curable-conducting polyurethanes. Prog. Org. Coat. 2016, 94, 79-89. [CrossRef]

7. Yun, S.; Im, H.; Kim, J. The effect of different hard segments in polyurethane on the electrical conductivity of polyurethane grafted multi-walled carbon nanotube/polyurethane nanocomposites. Synth. Met. 2011, 161, 1361-1367. [CrossRef]

8. Wang, F.F.; Feng, L.J.; Li, G.Z. Properties of waterborne polyurethane conductive coating with low MWCNTs content by electrostatic spraying. Polymers 2018, 10, 1406. [CrossRef]

9. Huang, A.; Peng, X.; Turng, L. In-situ fibrillated polytetrafluoroethylene (PTFE) in thermoplastic polyurethane (TPU) via melt blending: Effect on rheological behavior, mechanical properties, and microcellular foamability. Polymers 2018, 134, 263-274. [CrossRef]

10. Wang, X.C.; Jing, X.; Peng, Y.Y.; Ma, Z.K.; Liu, C.T.; Turng, L.S.; Shen, C.Y. The effect of nanoclay on the crystallization behavior, microcellular structure, and mechanical properties of thermoplastic polyurethane nanocomposite foams. Polym. Eng. Sci. 2016, 56, 319-327. [CrossRef]

11. Hu, B.; Deng, J.; Zheng, H.; Yu, S.; Gao, C. Synthesis of chiral oligomer-grafted biodegradable polyurethanes and their chiral-dependent influence on bone marrow stem cell behaviors. Macromol. Rapid Commun. 2016, 37, 1331-1336. [CrossRef] [PubMed]

12. Honarkar, H. Waterborne polyurethanes: A Review. J. Disper. Sci. Technol. 2018, 39, 507-516. [CrossRef]

13. Zhang, Y.; Shao, L.; Dong, D.; Wang, Y. Enhancement of water and organic solvent resistances of a waterborne polyurethane film by incorporating liquid polysulfide. RSC Adv. 2016, 6, 17163-17171. [CrossRef]

14. Tong, Y.; Bohm, S.; Song, M. The capability of graphene on improving the electrical conductivity and anti-corrosion properties of Polyurethane coatings. Appl. Surf. Sci. 2017, 424, 72-81. [CrossRef]

15. Sung, Y.H.; Kim, Y.D.; Choi, H.J.; Shin, R.; Kang, S.; Lee, H. Fabrication of superhydrophobic surfaces with nano-in-microstructures using UV-nanoimprint lithography and thermal shrinkage films. Appl. Surf. Sci. 2015, 349, 169-173. [CrossRef]

16. $\mathrm{Xi}$, J.; Jiang, L. Biomimic superhydrophobic surface with high adhesive forces. Ind. Eng. Chem. Res. 2008, 47, 6354-6357. [CrossRef]

17. Iijima, M.; Hayakawa, M.; Tatami, J.; Nakamura, K.; Nagashima, S. Design of nanoscale structured composite particles through mechanical process for fabricating a powder layer with rapid drying properties. Chem. Eng. Sci. 2019, 203, 113-121. [CrossRef]

18. Simpson, J.T.; Hunter, S.R.; Aytug, T. Superhydrophobic materials and coatings: a review. Rep. Prog. Phys. 2015, 78, 086501. [CrossRef]

19. Feng, S.; Zhong, Z.; Wang, Y.; Xing, W.; Drioli, E. Progress and perspectives in PTFE membrane: Preparation, modification, and applications. J. Membr. Sci. 2018, 549, 332-349. [CrossRef]

20. Qiang, F.; Hu, L.L.; Gong, L.X.; Zhao, L.; Li, S.N.; Tang, L.C. Facile synthesis of super-hydrophobic, electrically conductive and mechanically flexible functionalized graphene nanoribbon/polyurethane sponge for efficient oil/water separation at static and dynamic states. Chem. Eng. J. 2018, 334, 2154-2166. [CrossRef]

21. Koti Reddy, C.; Shailaja, D. Improving hydrophobicity of polyurethane by PTFE incorporation. J. Appl. Polym. Sci. 2015, 132, 42779. [CrossRef]

22. Huang, C.; Cheng, Q. Learning from nacre: Constructing polymer nanocomposites. Compos. Sci. Technol. 2017, 150, 141-166. [CrossRef]

23. Liang, Y.; Ju, J.; Deng, N.; Zhou, X.; Yan, J.; Kang, W.; Cheng, B. Super-hydrophobic self-cleaning bead-like SiO2@PTFE nanofiber membranes for waterproof-breathable applications. Appl. Surf. Sci. 2018, 442, 54-64. [CrossRef] 
24. Abenojar, J.; Tutor, J.; Ballesteros, Y.; del Real, J.C.; Martínez, M.A. Erosion-wear, mechanical and thermal properties of silica filled epoxy nanocomposites. Compos. Part B Eng. 2017, 120, 42-53. [CrossRef]

25. Shin, M.S.; Lee, Y.L.; Rahman, M.M.; Kim, H.D. Synthesis and properties of waterborne fluorinated polyurethane-acrylate using a solvent-/emulsifier-free method. Polymers 2013, 54, 4873-4882. [CrossRef]

26. Krol, P.; Krol, B.; Stagraczynski, R.; Skrzypiec, K. Waterborne cationomer polyurethane coatings with improved hydrophobic properties. J. Appl. Polym. Sci. 2013, 127, 2508-2519. [CrossRef]

27. Serkis, M.; Spirkova, M.; Hodan, J.; Kredatusova, J. Nanocomposites made from thermoplastic waterborne polyurethane and colloidal silica. The influence of nanosilica type and amount on the functional properties. Prog. Org. Coat. 2016, 101, 342-349. [CrossRef]

28. Zhang, S.; Yu, A.; Liu, S.; Zhao, J.; Jiang, J.; Liu, X. Effect of silica nanoparticles on structure and properties of waterborne UV-curable polyurethane nanocomposites. Polym. Bull. 2012, 68, 1469-1482. [CrossRef]

29. Wang, F.F.; Feng, L.J.; Lu, M. Mechanical Properties of Multi-Walled Carbon Nanotube/Waterborne Polyurethane Conductive Coatings Prepared by Electrostatic Spraying. Polymers 2019, 11, 714. [CrossRef]

30. Roach, P.; Shirtcliffe, N.J.; Newton, M.I. Progress in superhydrophobic surface development. Soft Matter 2008, 4, 224-240. [CrossRef]

(C) 2019 by the authors. Licensee MDPI, Basel, Switzerland. This article is an open access article distributed under the terms and conditions of the Creative Commons Attribution (CC BY) license (http://creativecommons.org/licenses/by/4.0/). 